\title{
An Efficient and Complete Automatic System for Detecting Lung Module
}

\author{
S. Balakrishnan ${ }^{1 *}$, J. Janet ${ }^{1}$, K. Sujatha ${ }^{2}$ and S. Sheeba Rani ${ }^{3}$ \\ 'Department of Information Technology, Sri Krishna College of Engineering and Technology, Coimbatore - 641008, \\ Tamil Nadu, India; balkiparu@gmail.co, janetjude1@rediffmail.com \\ ${ }^{2}$ Department of Computer Science and Engineering, Sri Krishna College of Engineering and Technology, \\ Coimbatore -641008,Tamil Nadu, India; sujatha.ssps@gmail.com \\ ${ }^{3}$ Department of Electrical and Electronics Engineering, Sri Krishna College of Engineering and Technology, \\ Coimbatore-641008, Tamil Nadu, India; sheebaranis@skcet.ac.in
}

\begin{abstract}
Objectives: To make a fully automated algorithm that is based on simple and quick steps, which produces consistent output for the same inputs. Methods/Statistical Analysis: For thorax and lung segmentation, region growing based method is used to segment the region of interest. The missing parts of the lungs are reconstructed using morphological operations. After that, nodules are detected based on the features of the reconstructed image. Artificial Neural Network has been used for classifying the images. Findings: An aggregate of 100 pictures with determination of $512 \times 512$ pixels with eight bits for every shading channel are caught. $90 \%$ affectability was obtained with 0.05 false positives for each picture. Application/Improvements: This framework distinguishes the phase of lung malignancy. The outcomes demonstrate that the tumors are of various measurements. By estimating the measurements of the tumor the lung disease stage can be recognized precisely utilizing the proposed technique. The outcomes indicate great potential for lung growth identification at beginning time.
\end{abstract}

Keywords: Automatic System, Filter, Lung Cancer, Neural Network, Region Based Method

\section{Introduction}

Cancer alludes to the uncontrolled increase of a gathering of cells in a specific area of the body. Malignancy is a genuine sickness in which developments of cells, additionally called growths, frame in the body and murder ordinary body cells. A gathering of quickly partitioning cells may shape a bump or mass of additional tissue. These masses are normally alluded to as tumors. Growth cells are named threatening tumors. Bosom disease is any type of threatening tumor that creates from bosom cells. Essential signs to search for on account of bosom malignancy are groups of micro calcifications, masses, and structural bends. As of late, the frequency rate of bosom malignancy has extensively expanded ${ }^{1}$.
Mortality from lung growth is required to keep ascending, to wind up around 17 million worldwide in 2030. Early discovery of lung disease can build the shot of survival among individuals. There are numerous systems to analyze the lung malignancy, for example, "Chest Radiograph (X-beam), Computed Tomography (CT), Magnetic Resonance Imaging (MRI sweep) and Sputum Cytology". Be that as it may, a large portion of these procedures are costly and tedious. In this manner, "there is an awesome requirement for another innovation to analyze the lung tumor in its beginning times". Image processing procedures give a decent quality instrument to enhancing the manual examination. The lungs are a couple of wipe like, cone-molded organs. The correct lung has three flaps, and is bigger than the left lung, which has

*Author for correspondence 
two projections. Lung malignancy is a malady of irregular cells duplicating and developing into a knob.

The lung growth is the second most regular tumor in the two men and ladies in Europe and in the United States that speaks to a noteworthy financial issue for medicinal services framework. In the report of American Cancer Society distributed in 2003 13\% of all malignancy determination is lung growth has been broke down in 5 years. Now and then rate increments up to $49 \%$ and when sickness is as yet restricted and furthermore perceived. Lung disease is caused by wild unpredictable development of cells in lung tissue. These lung tissue variations from the norm are frequently called lung knobs. They are little and generally round mass of tissue around $1 \mathrm{~mm}$ to $30 \mathrm{~mm}$ in measure. As a rule, they can be ordered into 4 bunches including juxta-vascular, very much encompassed pleural tail, and juxtapleural Pulmonary knobs are the portrayal of the beginning period of the lung disease. Also, this can be identified utilizing CT filter, MRI, SPECT and PET. PC Tomography (CT) $t$ is said to be the best imaging methodology for location of aspiratory knobs especially, since the presentation of winding (helical) innovation. The CT affectability increments with a lessening of the cut thickness because of an abatement of fractional volume impact, and covering pictures reproduction enhances the recognition of little knobs situated at the limit of two bordering non-covering pictures. At first there are different preparing procedures which are connected to remove the influenced district of lung from the CT pictures. At that point with a bunching calculation the division procedure is performed. This paper goes for a correlation of various techniques in lung knob recognition by utilizing CT. A neural framework approach for recognizing contender laid out wounds in digitized mammograms is $\mathrm{in}^{2}$. The neural framework arranged using back spread figuring's. The framework depends dominatingly on the noteworthy difference between the histogram of the standard tissue and that of the cancer-causing tissue. "Single and multi scale distinguishing proof of masses in mechanized mammograms" is considered in ${ }^{3}$. Scale is a "crucial issue in the modernized disclosure of masses in mammograms, due to the extent of possible sizes masses can have". In this work, it was examined if distinguishing proof of masses ought to be conceivable at a lone scale, or whether it is all the more fitting to use the yield of the acknowledgment strategy at different scales in a multi scale plot.

$\mathrm{In}^{4}$ utilized lung CT pictures separated from NIH/NCI Lung Database Consortium and proposed a programmed
PC helped diagnosing framework for recognition of lung tumor by examining these lung CT pictures. The creators of the paper have utilized a few stages for the identification of lung malignancy. Right off the bat, they removed the lung district from the PC tomography picture utilizing different picture handling procedures, for example, bit picture cutting, disintegration and wiener channel. In the initial step the bit picture cutting system was utilized to change over the CT pictures into a parallel picture then after extraction the locale developing division calculation was utilized for portioning the extricated lung areas. After division of lung district they utilized control based model to group the malignancy knobs. At last, "an arrangement of analysis rules was produced from the removed highlights and with the assistance of diagnostics pointer". It was watched that the proposed technique accomplished the general exactness of $80 \%$.

$\mathrm{In}^{\frac{5}{5}}$ has built up a mechanized framework, that was identified the lung knobs with the assistance of CT filter pictures. The mechanized framework $\mathrm{k}^{6} \underline{-10}$ comprises of two phases, initial one is lung division and upgrade and second one is include extraction and order. For evacuating foundation and concentrates the knobs from a picture, the limit division procedure was connected. Whenever extraction and division were finished, at that point a component vector was utilized to- ascertain the anomalous area. After that the areas were ordered utilizing neuro fluffy classifier. This framework gives the office to distinguish the littlest knobs which prompt early analysis of lung disease. In ${ }^{11}$ explained an automatic Computer Aided Diagnosis (CAD) system that includes three steps such as thresholding the CT image by segmentation, labeling the founded regions and regions are extracted for further analysis for the "detection of lung cancer using CT scan images".

\section{Methods Used}

\subsection{Hybrid Median Filter}

Impulse noise reduction or removal is an exceptionally dynamic research zone of picture handling. A nonlinear cross breed channel for expelling settled drive commotion clamor from shading pictures has been proposed in this examination. System depends on scientific morphology and trimmed standard middle channel. Half and half middle channel is windowed channel of nonlinear class that effortlessly evacuates drive clamor while protect- 
ing edges. In examination with fundamental rendition of the middle channel cross breed one has better corner safeguarding attributes. The fundamental thought behind channel is for any component of the flag (picture) applies middle method a few times changing window shape and after that take the middle of the got middle qualities.

\subsection{Image Contrast Improvement using} AHE

A many-sided quality difference in picture is expert by Adaptive histogram evening out. AHE is a PC picture dealing with strategy used to improve separate in pictures. It differs from standard histogram evening out in the respect that the flexible system figures a couple of histograms, each contrasting with a specific region of the photo, and utilizations them to redistribute the daintiness estimations of the photo. It is in this way suitable for improving the close-by unpredictability and redesigning the implications of edges in each district of a photo. In any case, AHE tends to over increase tumult in by and large homogeneous regions of a photo.

\subsection{Region Growing Segmentation}

Locale developing is a basic district based picture division strategy. It is additionally named a pixel-based picture division strategy since it includes the choice of beginning seed focuses. This way to deal with division inspects neighboring pixels of introductory seed focuses and decides if the pixel neighbors ought to be added to the area. The procedure is iterated on, in an indistinguishable way from general information bunching calculations. Figure 1 shows the Example of region growing.

\section{Proposed System}

Image acquisition is a process of getting an input image which may be Healthy lung image or Lung Nodule Image for the process of Nodule detection using Image Processing Algorithms. "Pre-processing is a common name for operations with the images at the lowest level of abstraction both input and output is the input images". The point of pre-handling is a change of picture information that smothers undesirable picture information mutilations or upgrades the some picture highlights imperative for the further preparing. Pre-preparing strategy comprise of Resizing, Noise Removal, Color Transformation for additionally procedure of location of Lung Nodule.

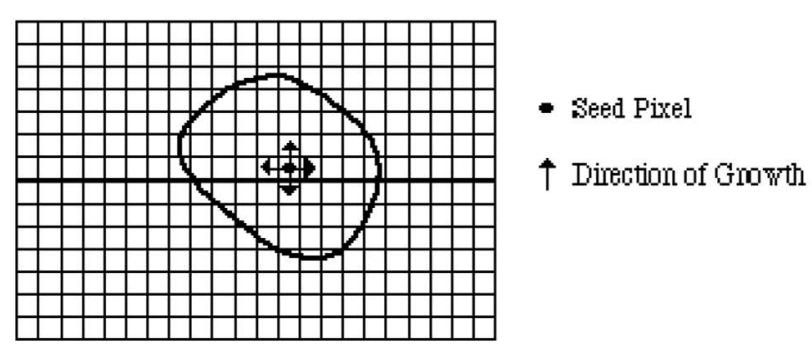

(a) Stait of Growing a Region

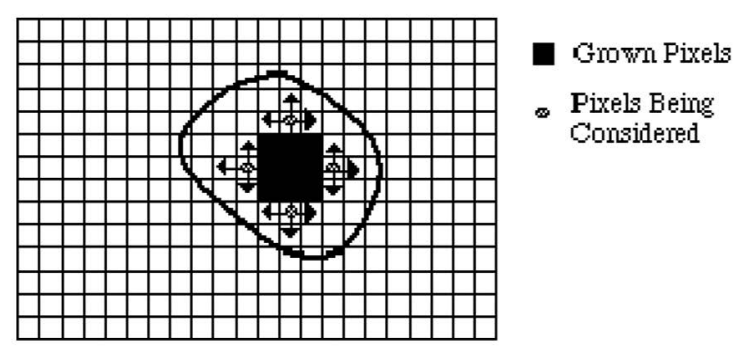

(b) Growing Process After a Few Iterations

Figure 1. Example of region growing.

Four classifications of picture pre-handling techniques as indicated by the "span of pixel neighborhood that is utilized for the count of new pixel brilliance: (I) Pixel splendor changes (ii) Geometric changes (iii) Pre-preparing strategies that utilization a nearby neighborhood of the handled pixel, (iv) Image reclamation that requires information about the whole picture". In machine learning, "design acknowledgment and in picture preparing, highlight extraction begins from an underlying arrangement of estimated information and fabricates determined esteems (highlights) planned to be educational and non-repetitive, encouraging the resulting learning and speculation steps, and at times prompting better human elucidations". Lung Cancer Detection System Process is given in the Figure 2.

\section{Experimental Results}

For experimentation of the proposed strategy, the "CT pictures are acquired from a NIH/NCI Lung Image Database Consortium (LIDC) dataset that gives the opportunity to do the recommended examine". All in all, the "middle channel permits a lot of high spatial recurrence detail to pass while staying exceptionally viable at expelling clamor on pictures were not as much as half of the pixels in a smoothing neighborhood have been influenced". $\mathrm{B}=$ medfilt $2(\mathrm{~A},[\mathrm{~m}, \mathrm{n}])$ performs middle sifting of the lattice An of every two measurements. Each 
"yield pixel contains the middle an incentive in the $\mathrm{m} \times \mathrm{n}$ neighborhood around the relating pixel in the picture". Medfilt2 cushions the "picture with 0's on the edges, so the middle esteems for focuses inside one-a large portion

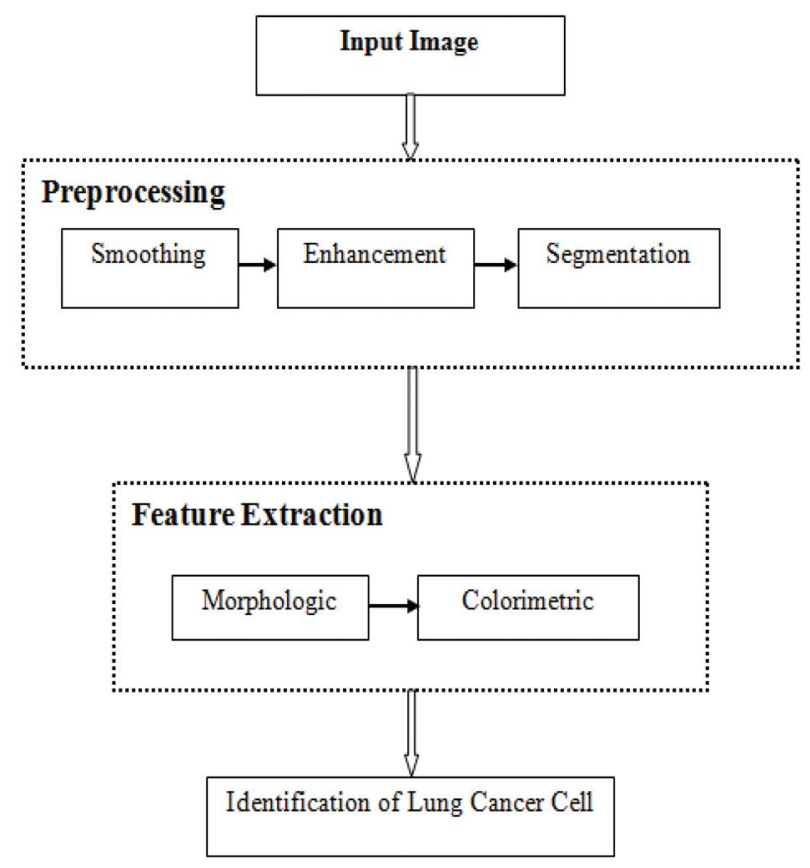

Figure 2. Lung cancer detection system process. of the width of the area $([\mathrm{m}, \mathrm{n}] / 2)$ of the edges may seem mutilated as appeared in Figure 3 (b)". In this framework, "client have built up a programmed CAD framework for early discovery of lung disease utilizing Lung CT pictures in which an abnormal state of affectability has been accomplished, with a sensible measure of false positives per picture, (90\% affectability with 0.05 false positives for each picture)" the radiologist's conclusion. The Figure 4 shows the detection of lung cancer.

\section{Conclusion}

Lung danger is a champion among the most dangerous diseases on the planet. Redress Diagnosis and early area of lung infection can construct the survival rate. The present procedures join examination of X-bar, CT channel, MRI, PET pictures. The ace specialists break down the affliction and recognize the period of development by experience. The treatment fuses therapeutic technique, chemotherapy, radiation treatment and concentrated on treatment. These solutions are broad, costly and unbearable. Consequently, an undertaking is made to atomize this strategy to perceive the lung ailment using picture taking care of frameworks.

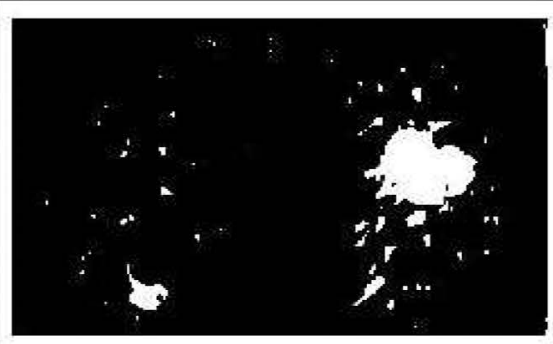

(a) Nodule Reconstruction

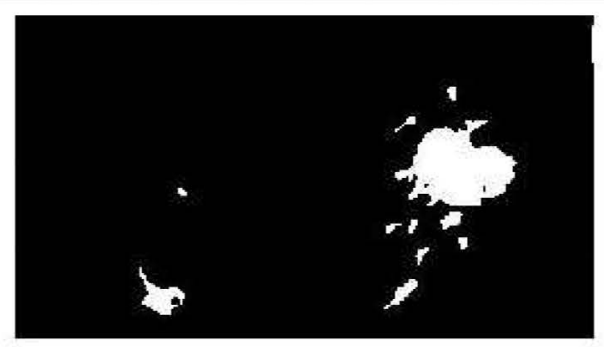

(b) Removing Small Componets

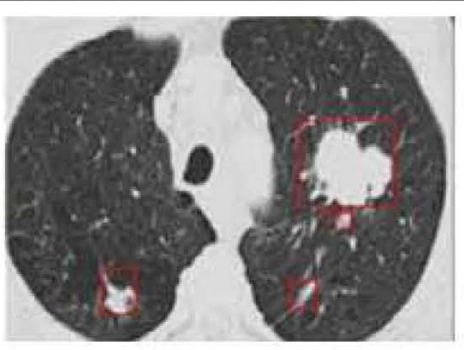

(c) Final Image

Figure 3. Stages of lung cancer detection.

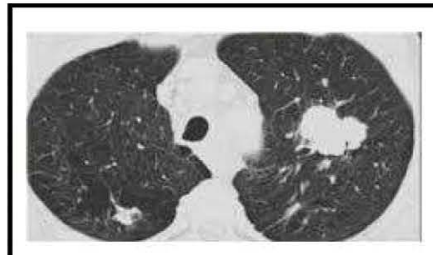

(a) Input Image

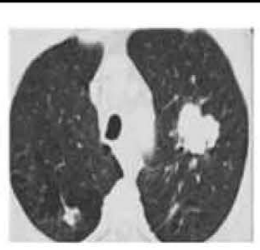

(b) Noise removal

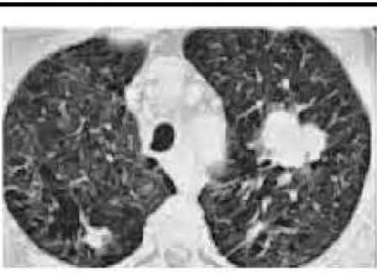

(c) Enhanced Image

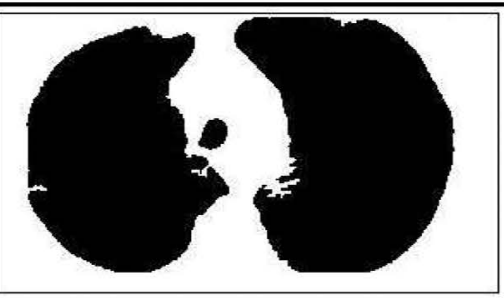

(d) Thorax Segmentation

Figure 4. Detection of lung cancer. 
CT channel pictures are acquired from various mending offices. These photos consolidate fewer hullabaloos when diverged from X-bar and MRI pictures. A photo change methodology is creating for earlier disease acknowledgment and treatment orchestrates; the time factor is considered to discover the irregularity issues in target pictures. The CT got pictures are taken care of. The area of interest i.e., tumor is recognized correctly from the main picture.

\section{References}

1. Palanikumar P, Shirly SG, Balakrishnan S. An effective two way classification of breast cancer images. International Journal of Applied Engineering Research. 2015; 10(21):42472-5.

2. Youssry N, Abou-Chadi FE, El-Sayad AM. A neural network approach for mass detection in digitized mammograms. ACBME. 2002.

3. Brake GM, Karssemeijer N. Single and multi scale detection of masses in digital mammograms. IEEE Transactions on Medical Imaging. 1999; 18(7):628-39. PMid:10504096 Crossref.

4. Sharma D, Jindal G. Identifying lung cancer using image processing techniques. International Conference on Computational Techniques and Artificial Intelligence (ICCTAI'2011). 2011; p. 872-80.
5. Tariq A, Akram MU, Javed MY. Lung nodule detection in CT images using neuro fuzzy classifier. In Computational Intelligence in Medical Imaging (CIMI). IEEE 4th International Workshop. 2013; p. 49-53. Crossref.

6. Chan HP, Doi K, Vyborny CJ, Lam KL, Schmidt RA. Computer-aided detection of micro calcifications in mammograms methodology and preliminary clinical study. Investigative Radiol. 1998; 23:664-71.

7. Karssemeijer N. Biomedical image processing and biomedical visualization. Proc SPIE; 1993. p. 776-86. Crossref.

8. Nakayama R, Uchiyama Y. Development of new filter bank for detection of nodular patterns and linear patterns in medical images. Systems and Computers in Japan. 2005; 36(13):81-91. Crossref.

9. Penedo MG, Carreira MJ, Mosquera A, Cabello D. Computeraided diagnosis: a neural network- based approach to lung nodule detection. IEEE Transactions on Medical Imaging. 1998; 17:872-80. PMid:10048844 Crossref.

10. Kanazawa K, Kubo M, Niki N. Computer aided diagnosis system for lung cancer based on helical CT images. Proceedings of the 13th International Conference on Pattern Recognition. 1996; p. 381-5. Crossref.

11. Amjed S, Jaber EB. Automated detection of lung cancer using statistical and morphological image processing techniques. Journal of Biomedical Graphics and Computing. 2014; 4(2):1-10. 